Internat. J. Math. \& Math. Sci.

Vol. 22, No. 3 (1999) 637-642

S $0161-1712\langle 99\rangle 22637-3$

(c) Electronic Publishing House

\title{
ON THE VERTICAL BUNDLE OF A PSEUDO-FINSLER MANIFOLD
}

\author{
AUREL BEJANCU and HANI REDA FARRAN
}

(Received 21 August 1996 and in revised form 24 November 1997)

\begin{abstract}
We define the Liouville distribution on the tangent bundle of a pseudo-Finsler manifold and prove that it is integrable. Also, we find geometric properties of both leaves of Liouville distribution and the vertical distribution.
\end{abstract}

Keywords and phrases. Pseudo-Finsler manifold, Liouville distribution, vertical bundle.

1991 Mathematics Subject Classification. 53B50, 53B30.

1. Introduction. As it is well known, the vertical vector bundle VTM of a manifold $M$ is an integrable distribution on the tangent bundle $T M$ of $M$. The present paper is concerned with the study of the geometry of leaves of VTM in the case when the base manifold $M$ carries a pseudo-Finsler structure. In our study, the pseudo-Finsler metric is, in fact, considered as a semi-Riemannian metric on VTM. This enables us to define the Liouville distribution which will be the important tool in studying the leaves of VTM.

The main results are stated in Theorems 3.2 and 3.3.

2. Preliminaries. Let $M$ be a smooth ( $C^{4}$ is enough) $m$-dimensional manifold and $T M$ be the tangent bundle of $M$. Denote by $\Theta$ the zero section of $T M$ and set $T M^{\prime}=$ $T M \backslash \Theta(M)$. The coordinates of a point of $T M$ are denoted by $\left(x^{i}, y^{i}\right)$, where $\left(x^{i}\right)$ and $\left(y^{i}\right)$ are the coordinates of a point $x \in M$ and the components of a vector $y$ in $T_{x} M$, respectively. Consider a continuous function $L(x, y)$ defined on $T M$ and suppose that the following conditions are satisfied.

$\left(L_{1}\right) L$ is smooth on $T M^{\prime}$.

$\left(L_{2}\right) L$ is positive homogeneous of degree two with respect to $y$, i.e., we have

$$
L(x, k y)=k^{2} L(x, y) \quad \forall k>0 .
$$

$\left(L_{3}\right)$ The metric tensor

$$
g_{i j}(x, y)=\frac{1}{2} \frac{\partial^{2} L}{\partial y^{i} \partial y^{j}}
$$

has $q$ negative eigenvalues and $m-q$ positive eigenvalues for all $(x, y) \in T M^{\prime}$.

Then we say that $F^{m}=(M, L)$ is a pseudo-Finsler manifold of index $q$. If, in particular, $q=0, F^{m}$ becomes a Finsler manifold (cf. Rund [9, p. 5]).

Denote by $V T M^{\prime}$ the vertical vector bundle over $T M^{\prime}$, that is, $V T M^{\prime}=\operatorname{ker} d \pi$, where $\pi: T M^{\prime} \rightarrow M$ is the canonical projection and $d \pi$ is its differential mapping. Then any section of $V T M^{\prime}$ is a Finsler vector field. Also, any section of the dual vector bundle 
$V^{*} T M^{\prime}$ is a Finsler 1-form. In this way, the entire Finsler tensor calculus can be developed via the vertical vector bundle (cf. Bejancu [6]).

Denote by $F\left(T M^{\prime}\right)$ the algebra of smooth functions on $T M^{\prime}$ and by $\Gamma\left(V T M^{\prime}\right)$ the $F\left(T M^{\prime}\right)$-module of smooth sections of $V T M^{\prime}$. We keep the same notation for any other vector bundle. We also use the Einstein convention, that is repeated indices with one upper index and one lower index denote summation over their range.

Let $U^{\prime}$ be a coordinate neighborhood of $T M^{\prime}$ and $X \in \Gamma\left(V T M_{\mid U^{\prime}}^{\prime}\right)$. As $V T M^{\prime}$ is an integrable distribution on $T M^{\prime}$, it follows that $\left\{\partial / \partial y^{i}\right\}$ is a basis of $\Gamma\left(V T M_{\mid U^{\prime}}^{\prime}\right)$, and thus $X=X^{i}(x, y)\left(\partial / \partial y^{i}\right)$. An important Finsler vector field is defined by

$$
V=y^{i} \frac{\partial}{\partial y^{i}},
$$

and it is called the Liouville vector field.

Next, we denote by $T_{2}^{0}\left(V T M^{\prime}\right)$ the vector bundle over $T M^{\prime}$ of all bilinear mappings on $V T M^{\prime}$. Then, from (2.2), $\left\{g_{i j}\right\}$ define a global section of $T_{2}^{0}\left(V T M^{\prime}\right)$ given on $U^{\prime}$ by

$$
g(X, Y)(x, y)=g_{i j}(x, y) X^{i}(x, y) X^{j}(x, y) \quad \forall X, Y \in \Gamma\left(V T M^{\prime}\right) .
$$

As $g$ is symmetric, condition $\left(L_{3}\right)$ enables us to claim that $g$ is a semi-Riemannian metric of index $q$ on $V T M^{\prime}$ (cf. O'Neill [8]). Throughout the paper, we suppose that $V$ is space-like with respect to $g$, i.e., we have

$$
g(V, V)>0 .
$$

By using the homogeneity of $L$, we deduce that

$$
L(x, y)=g_{i j}(x, y) y^{i} y^{j} .
$$

Thus, taking account of (2.4) and (2.5) in (2.6), we obtain $L>0$ on $T M^{\prime}$. The fundamental function of $F^{m}$ (cf. Matsumoto [7, P. 101]) is defined by $F=L^{1 / 2}$, and thus it is positive homogeneous of degree one with respect to $y$. Hence, we have (cf. BaoChern [1])

$$
y^{i} \frac{\partial F}{\partial y^{i}}=F
$$

and

$$
y^{i} \frac{\partial^{2} F}{\partial y^{i} \partial y^{j}}=0
$$

Moreover, since $L=F^{2}$, from (2.6), we get

$$
g_{i j}=F \frac{\partial^{2} F}{\partial y^{i} \partial y^{j}}+\frac{\partial F}{\partial y^{i}} \frac{\partial F}{\partial y^{j}} .
$$

By contracting (2.9) by $y^{j}$ and taking account of (2.7) and (2.8), we obtain

$$
g_{i j} y^{j}=F \frac{\partial F}{\partial y^{i}} \text {. }
$$

Similarly, since $L$ is positive homogeneous of degree two with respect to $y$, we have

$$
y^{i} \frac{\partial L}{\partial y^{i}}=2 L
$$


which implies that

$$
y^{i} \frac{\partial^{2} L}{\partial y^{i} \partial y^{j}}=\frac{\partial L}{\partial y^{j}} .
$$

Finally, differentiating (2.12) with respect to $y^{k}$, we obtain

$$
y^{i} \frac{\partial^{3} L}{\partial y^{i} \partial y^{j} \partial y^{k}}=0
$$

which yields

$$
\frac{\partial g_{i j}}{\partial y^{k}} y^{i}=0, \quad \frac{\partial g_{i j}}{\partial y^{k}} y^{j}=0, \quad \frac{\partial g_{i j}}{\partial y^{k}} y^{k}=0 .
$$

Next, we define $\xi=(1 / F) V$ and by using (2.3), (2.4), and (2.6), we get

$$
g(\xi, \xi)=1 \text {. }
$$

By means of $g$ and $\xi$, we define the Finsler 1 -form $\eta$ by

$$
\eta(X)=g(X, \xi) \quad \forall X \in \Gamma\left(V T M^{\prime}\right) .
$$

Denote by $\{\xi\}$ the line vector bundle over $T M^{\prime}$ spanned by $\xi$ and define the Liouville distribution as the complementary orthogonal distribution $S T M^{\prime}$ to $\{\xi\}$ in $V T M^{\prime}$ with respect to $g$. Hence, $S T M^{\prime}$ is defined by $\eta$, that is we have

$$
\Gamma\left(S T M^{\prime}\right)=\left\{X \in \Gamma\left(V T M^{\prime}\right) ; \eta(X)=0\right\} .
$$

Thus, any Finsler vector field $X$ can be expressed as follows:

$$
X=P X+\eta(X) \xi
$$

where $P$ is the projection morphism of $V T M^{\prime}$ on $S T M^{\prime}$. By direct calculations, we obtain

$$
g(X, P Y)=g(P X, P Y)=g(X, Y)-\eta(X) \eta(Y) \quad \forall X, Y \in \Gamma\left(V T M^{\prime}\right) .
$$

Denote by $\left\{w^{i}\right\}$ the dual basis in $\Gamma\left(\left.V^{*} T M^{\prime}\right|_{U^{\prime}}\right)$ with respect to $\left\{\partial / \partial y^{i}\right\}$. Then the local components of $\eta$ and $P$ with respect to the basis $\left\{w^{i}\right\}$ and $\left\{w^{i} \otimes \partial / \partial y^{j}\right\}$, respectively, are given by

$$
\eta_{i}=\frac{\partial F}{\partial y^{i}}
$$

and

$$
P_{i}^{j}=\delta_{i}^{j}-\frac{1}{F} \eta_{i} y^{j},
$$

where $\delta_{i}^{j}$ are the components of the Kronecker delta.

The indicatrix of $F^{m}$ is the hypersurface $I$ of $T M^{\prime}$ given by the equation $F(x, y)=1$.

More about pseudo (indefinite)-Finsler manifolds can be found in a series of papers by Beem [2, 3, 4, 5].

3. Geometry of the vertical vector bundle of $F^{m}$ via the Liouville distribution. In this section, we suppose that $F^{m}$ is a pseudo-Finsler manifold which is never a semi-Riemannian manifold, that is $\left\{g_{i j}\right\}$ does not depend on $\left(x^{i}\right)$ alone.

First, we prove the following result. 
THEOREM 3.1. Let $F^{m}=(M, L)$ be a pseudo-Finsler manifold. Then the Liouville distribution of $F^{m}$ is integrable.

Proof. Let $X, Y \in \Gamma\left(S T M^{\prime}\right)$. As $V T M^{\prime}$ is an integrable distribution on $T M^{\prime},[X, Y]$ lies in $\Gamma\left(V T M^{\prime}\right)$. Hence, we need only to show that $[X, Y]$ has no component with respect to $\xi$. By using (2.4) and (2.16), we deduce that $X \in \Gamma\left(S T M^{\prime}\right)$ if and only if

$$
g_{i j}(x, y) y^{i} X^{j}(x, y)=0,
$$

where $X^{j}$ are the components of $X$. Differentiate (3.1) with respect to $y^{k}$ and use (2.14) to obtain

$$
g_{k j}(x, y) X^{j}(x, y)+g_{i j}(x, y) y^{i} \frac{\partial X^{j}}{\partial y^{k}}(x, y)=0 .
$$

Then by direct calculations using (2.3), (2.4), and (3.2), we infer that

$$
g([X, Y], \xi)=\frac{1}{F} g_{i j} y^{i}\left\{\frac{\partial Y^{j}}{\partial y^{k}} X^{k}-\frac{\partial X^{j}}{\partial y^{k}} Y^{k}\right\}=0
$$

which completes the proof.

Based on the above results, we may say that the geometry of the leaves of $V T M^{\prime}$ should be derived from the geometry of the leaves of $S T M^{\prime}$ and of integral curves of $\xi$. In order to get this interplay, we consider a leaf $N^{\prime}$ of $V T M^{\prime}$ given locally by $x^{i}=a^{i}, i \in\{1, \ldots, m\}$, where the $a^{i}$ 's are constants. Then, from (2.2), $g_{i j}(a, y)$ are the components of a semi-Riemannian metric $g$ of index $q$ on $N^{\prime}$. Denote by $\nabla$ the Levi-Civita connection on $N^{\prime}$ with respect to $g$ and consider the Christoffel symbols $C_{i j}^{k}$ of $\nabla$. By using (2.2) and the usual formula for $C_{i j}^{k}$ (see O’Neill [8, P. 62]), we obtain

$$
C_{i j}^{k}(a, y)=\frac{1}{2} g^{k h}(a, y) \frac{\partial g_{h i}}{\partial y^{j}}(a, y),
$$

where $\left\{g^{k h}(a, y)\right\}$ are the entries of the inverse matrix of the $m \times m$ matrix $\left[g_{k h}(a, y)\right]$. Contracting (3.4) by $y^{j}$, we deduce that

$$
C_{i j}^{k}(a, y) y^{j}=0 .
$$

By straightforward calculations using (3.5), (2.3), (2.19), (2.20), and (2.21), we obtain the covariant derivatives of $\xi, \eta$, and $P$ in the following lemma:

LEMмA 3.1. Let $F^{m}=(M, L)$ be a pseudo-Finsler manifold. Then, on any leaf $N^{\prime}$ of $V T M^{\prime}$, we have

$$
\begin{gathered}
\nabla_{X} \xi=\frac{1}{F} P X, \\
\left(\nabla_{X} \eta\right) Y=\frac{1}{F} g(P X, P Y),
\end{gathered}
$$

and

$$
\left(\nabla_{X} P\right) Y=-\frac{1}{F}\{g(P X, P Y) \xi+\eta(Y) P X\}
$$

for any $X, Y \in \Gamma\left(T N^{\prime}\right)$.

Now, we state the main results of the paper. 
THEOREM 3.2. Let $F^{m}=(M, L)$ be a pseudo-Finsler manifold and $N^{\prime}, N$, and $C$ be a leaf of $V T M^{\prime}$, a leaf of $S T M^{\prime}$ that lies in $N^{\prime}$, and an integral curve of $\xi$, respectively. Then we have the following assertions. (i) $C$ is a geodesic of $N^{\prime}$ with respect to $\nabla$.

(ii) $N$ is totally umbilical immersed in $N^{\prime}$.

(iii) $N$ lies in the indicatrix of $F^{m}$ and has constant mean curvature equal to -1 .

Proof. Replace $X$ by $\xi$ in (3.6) and obtain (i). Taking into account that $\xi$ is the unit normal vector field of $N$, the second fundamental form $B$ of $N$ as a hypersurface of $N^{\prime}$ is given by

$$
B(X, Y)=g\left(\nabla_{X} Y, \xi\right) \quad \forall X, Y \in \Gamma(T N) .
$$

On the other hand, by using (3.6) and taking into account that $g$ is parallel with respect to $\nabla$, we deduce that

$$
g\left(\nabla_{X} Y, \xi\right)=-\frac{1}{F} g(X, Y) \quad \forall X, Y \in \Gamma(T N) .
$$

Hence,

$$
B(X, Y)=-\frac{1}{F} g(X, Y),
$$

that is, $N$ is totally umbilical immersed in $N^{\prime}$. Now, from (2.10), it follows that

$$
\frac{y^{i}}{F}=g^{i j} \frac{\partial F}{\partial y^{j}}
$$

which proves that $\xi$ is a unit normal vector field for both $N$ and the component $I_{a}$ of the indicatrix $I$ at $a \in M$. Thus, $N$ lies in $I_{a}$ and $F(a, y)=1$ at any point $y \in N$. Then (3.11) becomes

$$
B(X, Y)=-g(X, Y)
$$

which implies that

$$
\frac{1}{m-1} \sum_{i=1}^{m-1} \varepsilon_{i} B\left(E_{i}, E_{i}\right)=-1,
$$

where $\left\{E_{i}\right\}$ is an orthonormal frame field on $N$ of signature $\left\{\varepsilon_{i}\right\}$. Hence, the mean curvature of $N$ is -1 . The proof is complete.

THEOREM 3.3. Let $F^{m}=(M, L)$ be a pseudo-Finsler manifold and $N^{\prime}$ be a leaf of the vertical vector bundle $V T M^{\prime}$. Then the sectional curvature of any nondegenerate plane section on $N^{\prime}$ containing the Liouville vector field is equal to zero.

Proof. Denote by $R^{\prime}$ the curvature tensor field of $\nabla$ on $N^{\prime}$. Then, by using (3.6) and (3.8), we obtain

$$
R^{\prime}(X, \xi) \xi=\frac{1}{F^{2}}(1-\xi(F)) P X
$$

for any unit vector field $X$ on $N^{\prime}$. But from (2.3) and (2.7), we deduce that $\xi(F)=1$. Hence, the sectional curvature of a plane section $\{X, \xi\}$ vanishes on $N^{\prime}$.

CORROLARY 3.1. Let $F^{m}=(M, L)$ be a pseudo-Finsler manifold. Then there exist no leaves of $V T M^{\prime}$ which are positively or negatively curved. 


\section{REFERENCES}

[1] D. Bao and S. S. Chern, On a notable connection in Finsler geometry, Houston J. Math. 19 (1993), no. 1, 135-180. MR 94g:53049. Zbl 787.53018.

[2] J. K. Beem, Indefinite Fisler spaces and timelike spaces, Canad. J. Math. 22 (1970), 10351039. MR 42\#2415. Zbl 204.22003.

[3] _ Motions in two dimensional indefinite Finsler spaces, Indiana Univ. Math. J. 21 (1971/1972), 551-555. MR 45 1095. Zbl 226.53015.

[4] _ On the indicatrix and isotropy group in Finsler spaces with Lorentz signature, Atti Accad. Naz. Lincei, Rend. Cl. Sci. Fis. Mat. Natur. (8) 54 (1973), 385-392. MR 50 11074. Zbl 285.53023.

[5] _ Characterizin Finsler spaces which are pseudo-Riemannian of constant curvature, Pacific J. Math. 64 (1976), no. 1, 67-77. MR 54 8504. Zbl 326.53065.

[6] A. Bejancu, Finsler Geometry and Applications, Ellis Horwood Series: Mathematics and its Applications, Ellis Horwood, New York, 1990. MR 91i:53075. Zbl 702.53001.

[7] M. Matsumoto, Foundations of Finsler Geometry and Special Finsler Spaces, Kaiseisha Press, Shigaken, 1986. MR 88f:53111. Zbl 594.53001.

[8] B. O'Neill, Semi-Riemannian Geometry. With Applications to Relativity, Pure and Applied Mathematics, vol. 103, Academic Press, Inc., New York, London, 1983. MR 85f:53002. Zbl 531.53051.

[9] H. Rund, The Differential Geometry of Finsler Spaces, Die Grundlehren der Mathematischen Wissenschaften, vol. 101, Springer-Verlag, Berlin, Gottingen, Heidelberg, 1959. MR 21\#4462. Zbl 087.36604.

Bejancu: Department of Mathematics, Technical University of IASi, C. P. 17, IASi 1, 6600, IASI, ROMANIA

Current address: DePARTMENT OF MATHEMATICS AND COMPUTER SCIENCES, KUWAIT UNIVERSITY, P.O. BOX 5969, SAFAT 13060, KUWAIT

E-mail address: relu@math. tuiasi . ro and bejancu@math-1.sci.kuniv.edu.kw

FARRAN: DePARTMENT OF MATHEMATICS AND COMPUTER SCIENCES, KUWAIT UNIVERSity, P.O. BOX 5969, SAFAT 13060, KUWAIT

E-mail address: farranamath-1.sci.kuniv.edu.kw 


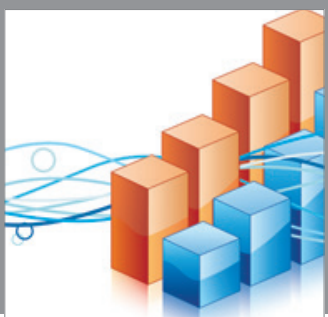

Advances in

Operations Research

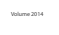

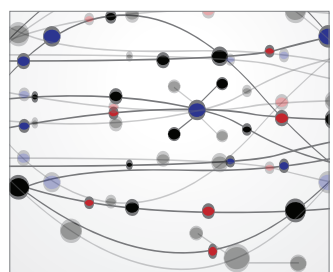

\section{The Scientific} World Journal
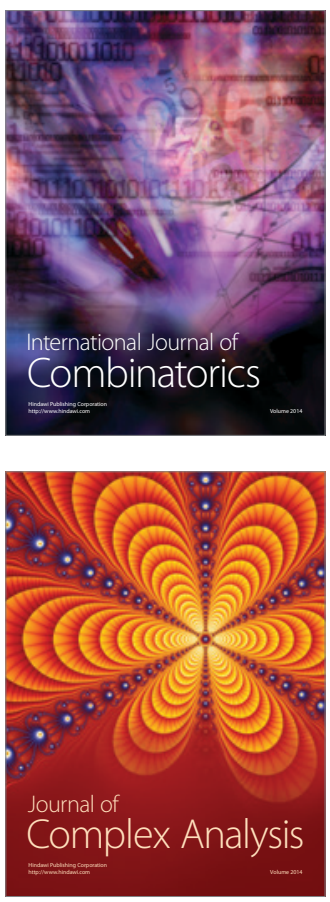

International Journal of

Mathematics and

Mathematical

Sciences
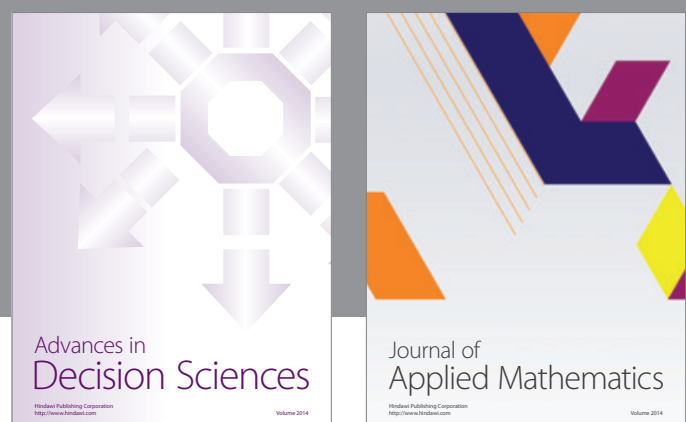

Journal of

Applied Mathematics
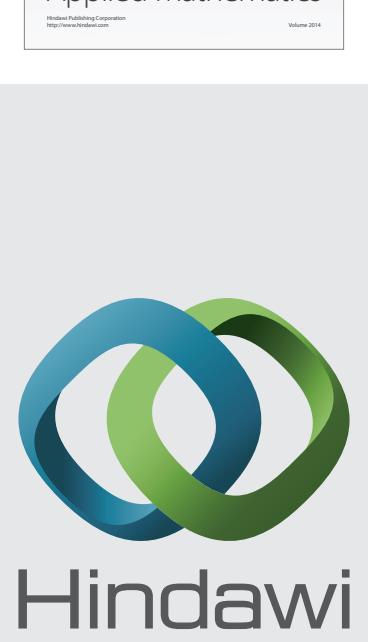

Submit your manuscripts at http://www.hindawi.com
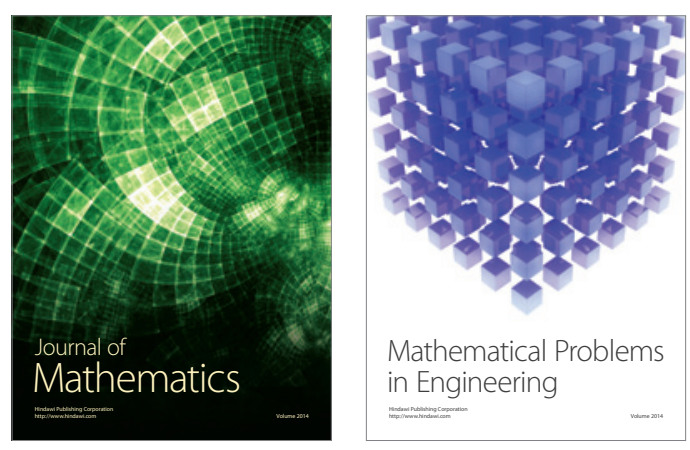

Mathematical Problems in Engineering
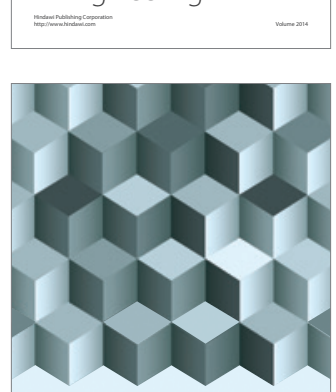

Journal of

Function Spaces
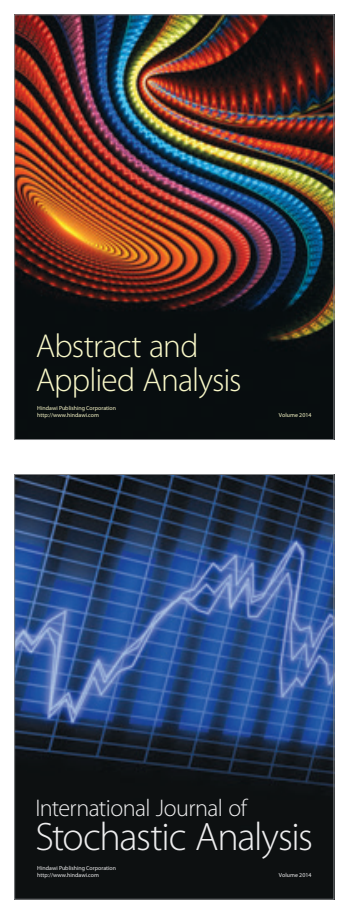

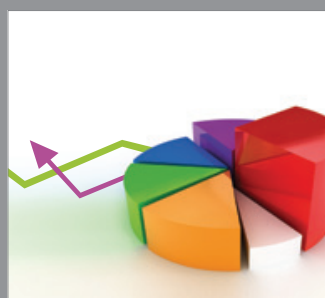

ournal of

Probability and Statistics

Promensencen
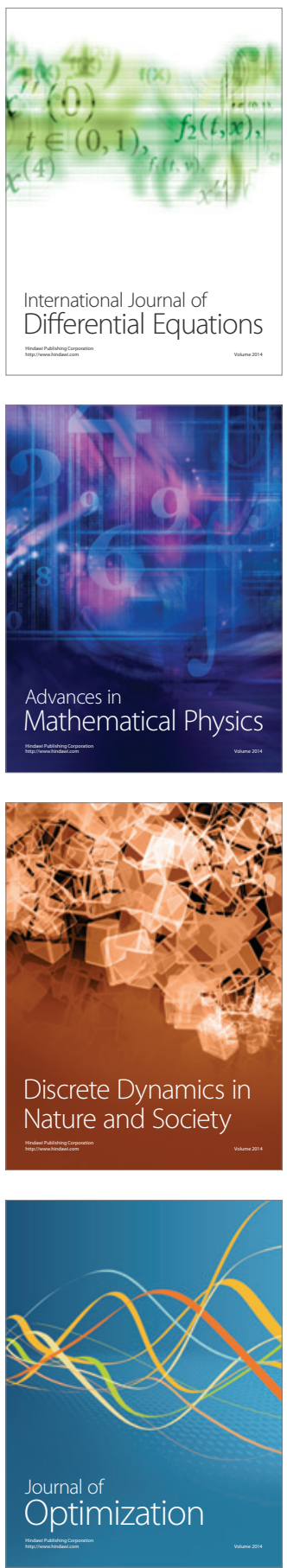